\title{
Dissecting the molecular mechanisms in craniofrontonasal syndrome: differential mRNA expression of mutant EFNB1 and the cellular mosaic
}

\author{
Ilse Wieland*,1, Roman Makarov ${ }^{1}$, William Reardon ${ }^{2}$, Sigrid Tinschert ${ }^{3,4}$, Alice Goldenberg ${ }^{5}$, \\ Patrick Thierry ${ }^{6}$ and Peter Wieacker ${ }^{1,7}$

\begin{abstract}
${ }^{1}$ Institut für Humangenetik, Otto-von-Guericke-Universität, Magdeburg, Germany; ${ }^{2}$ National Centre for Medical Carl Gustav Carus, Technische Universität (TU), Dresden, Germany; ${ }^{4}$ Institut für Medizinische Genetik, Charité - Universitätsmedizin, Berlin, Germany; ${ }^{5}$ Centre Hospitalier Universitaire de Rouen, Unité de Génétique Clinique, Hôpital Charles Nicolle, Rouen, France; ${ }^{6}$ Service de Pédiatrie, Centre Hospitalier Intercommunal de la Haute-Saône, Vesoul, France; ${ }^{7}$ Institut für Humangenetik, Westfälische Wilhelms-Universität, Münster, Germany
\end{abstract} \\ Genetics, Our Lady's Hospital for Sick Children, Dublin, Ireland; ${ }^{3}$ Institut für Klinische Genetik, Medizinische Fakultät
}

Craniofrontonasal syndrome (CFNS) is an X-linked malformation syndrome with variable phenotype that is caused by mutations in the ephrin-B1 gene (EFNB1). Over $50 \%$ of $E F N B 1$ mutations result in premature termination codons that may elicit mRNA degradation by the nonsense-mediated decay pathway. To assess the effects of various mutations at the transcript level, expression of EFNB1 mRNA was studied by RT-PCR in fibroblast cultures established from CFNS female patients. Compared to the wild-type and two missense mutation alleles, severe depletion of transcripts was observed for mutant alleles harbouring either splice site mutation c.407-2A $>T$ at the exon $2 / 3$ boundary or frameshift mutation c.377_384delTCAAGAAG in exon 2. In contrast, escape from mRNA decay was observed for mutation c.614_615deICT, which generates a premature termination codon close to the 3 '-end of the penultimate exon 4 disobeying the '50-55 bp' rule. These results suggest differential degradation of mutant EFNB1 transcripts by the nonsense-mediated mRNA decay pathway. Although the clinical phenotypes of the patients were not highly suggestive of a phenotype-genotype correlation, the two female patients were diagnosed with diaphragmatic hernia harbouring putative ephrin-B1 truncating mutations. Previously, disease manifestation in heterozygous females had been attributed mainly to cellular interference of divergent cell populations expressing wild-type or mutant EFNB1, depending on the pattern of $\mathrm{X}$-inactivation. Upon clonal expansion of patient cells with either the wild-type or mutant EFNB1 on the active $\mathrm{X}$-chromosome, we were able to separate mutant and wild-type EFNB1-expressing cells in vitro, further supporting the concept of cellular interference in CFNS.

European Journal of Human Genetics (2008) 16, 184-191; doi:10.1038/sj.ejhg.5201968; published online 28 November 2007

Keywords: CFNS; EFNB1; X-inactivation; RT-PCR; premature termination; NMD

*Correspondence: Dr I Wieland, Institut für Humangenetik, Otto-vonGuericke-Universität, Leipziger Strasse 44, Magdeburg D-39120, Germany. Tel: + 4939167 15381; Fax: + 4939167 15066; E-mail: ilse.wieland@medizin.uni-magdeburg.de Received 28 August 2007; revised 30 October 2007; accepted 31 October 2007; published online 28 November 2007
Introduction

Mutations in ephrin-B1 gene (EFNB1) (MIM 300035) are detected in the vast majority of patients with familial and sporadic craniofrontonasal syndrome (CFNS [MIM 304110]). CFNS is characterized by craniofrontonasal dysplasia including severe hypertelorism, depressed nasal 
bridge and bifid nasal tip, frontal bossing, coronal suture synostosis, corpus callosum agenesis, and occasionally cleft lip or palate. Further manifestations in heterozygous females frequently include asymmetry of the thoracic skeleton, pectoral muscle and breasts, grooved nails and thick wiry hair. ${ }^{1}$ CFNS is an X-linked condition with an unusual pattern of inheritance, as females are more severely affected than males. This paradoxical manifestation of CFNS in females $v s$ males led to the proposal of several inheritance scenarios including the possibility of interacting CFNS gene products from different cells in heterozygous females. ${ }^{2,3}$ After detecting the CFNS-causing gene, EFNB1, the model of cellular interference was proposed as the main mechanism responsible for disease manifestation in CFNS females. ${ }^{1,4}$ The EFNB1 gene encodes the transmembrane protein ephrin-B1, that functions in bi-directional cell signalling. ${ }^{5}$ As a ligand for Eph receptors, ephrin-B1 drives forward signalling in the opposing Eph-expressing cell. As a receptor, ephrin-B1 drives reverse signalling in the ephrin-B1-expressing cell. The highly complex Eph/ephrin system plays guiding roles in cell migration and pattern formation during developmental morphogenesis. ${ }^{6}$ The EFNB1 gene is located in the border region of chromosome Xq12 and Xq13.1 and is subjected to random X-inactivation in females. ${ }^{7}$ Because of the random $\mathrm{X}$-inactivation pattern, heterozygous females are expected to express the EFNB1 gene in a mosaic pattern, giving rise to two cell populations regarding ephrin-B1. ${ }^{4,8}$ Accordingly, the severity of disease manifestation in heterozygous females has been mainly attributed to cellular interference of divergent cell populations, particularly of those derived from the neural crest. ${ }^{9,10}$ Hence, CFNS has been interpreted as a novel type of neurocristopathy. ${ }^{1}$

EFNB1 contains 5 exons, among which exons 2 and 3 encode the extracellular ephrin domain. The missense mutations constitute about $42 \%$ of EFNB1 mutations. Most of them occur in exons 2 and 3, leading to substitutions of amino-acid residues important for receptor-ligand interaction and outside-in signalling. ${ }^{4,9,11,12}$ The major type of mutation, accounting for up to 55\% of EFNB1 mutations, consists of nonsense, frameshift and splice site mutations that lead to premature termination codons (PTCs). Gene deletions observed in a few patients definitely cause loss of EFNB1 gene function. ${ }^{4,12,13}$ For the recurrently observed splice site mutations at the exon $2 / 3$ junction as well as PTCs occurring in exons 1-4, loss of gene function has been proposed but has not been proven. ${ }^{9,11,12,14}$ In fact, it has been shown in experimental settings that truncated soluble ephrin-B1-Fc fusion proteins may act in a dominant-negative fashion. ${ }^{15-17}$ For several disease-causing genes, however, it has been demonstrated previously that truncating mutations were associated with markedly reduced transcript amounts. ${ }^{18,19}$ The instability of these aberrant transcripts was due to degradation by the nonsense-mediated mRNA decay (NMD) pathway. ${ }^{20} \mathrm{NMD}$ appears to be a general surveillance mechanism of eukaryotic cells to eliminate aberrant transcripts resulting from incorrect RNA processing. NMD primarily affects PTCs that occur in internal exons. ${ }^{20}$

In this study, we aimed to determine expression of EFNB1 transcripts from alleles containing missense mutations in exons 2 and 3, frameshift mutations in exons 2 and 4 and a splice site mutation at the splice donor site of intron 2 of the EFNB1 gene in primary fibroblasts derived from CFNS female patients.

\section{Methods \\ Patients and cell culture}

Informed consent was obtained from the patients or their parents. Primary fibroblast cultures of CFNS patients were established from skin biopsies (patients S16, S17, S18) or from tissue removed during surgical therapeutic interventions (patients F3, S33, S39). Cells were maintained under standard cell culture conditions and harvested for genomic DNA or total RNA isolation. Subclones of primary fibroblast cultures were established by gentle trypsin treatment of the primary cultures. About 500 cells were spread on tissue culture plates $(94 / 16 \mathrm{~mm})$ containing $10-\mathrm{ml}$ medium with $20 \%$ fetal calf serum (Invitrogen $\mathrm{GmbH}$, Karlsruhe, Germany). Cell clones were subcultured using cloning cylinders. Clonality of surviving subclones was determined by the X-inactivation assay as described below. Inhibition of NMD was performed in parallel cell cultures as described $^{21}$ using cycloheximide (Sigma-Aldrich GmbH, Taufkirchen, Germany) dissolved in solvent DMSO (SigmaAldrich) at concentrations of 50 and $100 \mu \mathrm{g} / \mathrm{ml}$ for $4 \mathrm{~h}$ and $1 \mathrm{mg} / \mathrm{ml}$ for $2 \mathrm{~h}$ or solvent alone (1\% DMSO).

\section{Mutation detection and X-inactivation assay}

Genomic DNA from cultured cells was isolated using standard protocols. Mutations were detected by direct sequencing of genomic DNA as described previously. ${ }^{4,11}$ The corresponding genomic mutations were confirmed by exon-specific PCR amplification and restriction enzyme digestion in all of the primary cell cultures. Primers and PCR conditions were as described. ${ }^{4}$ The PCR products were cleaved with restriction enzymes BslI detecting c.161C $>$ T, SfaNI for c.409A > G, HinfI for c.377_384del and c.614_615delCT, PstI for c.407-2A $>$ G and c.407-2A $>$ T (all enzymes were from New England Biolabs $\mathrm{GmbH}$, Frankfurt, Germany). The X-inactivation pattern in cultured cells was determined by the human androgen receptor X-inactivation (HUMAR) assay. ${ }^{22}$ Briefly, this assay uses a highly polymorphic CAG repeat in the androgen receptor gene. Methylation of this repeat correlates with $\mathrm{X}$-inactivation. Cleavage of the DNA with methylationsensitive restriction enzyme HpaII before PCR shows the inactivated X-chromosomes. PCR was performed using 
Cy5-labeled PCR primers (MWG Biotech, Ebersberg, Germany). PCR products were resolved on an ALFexpress sequencer (Amersham Biosciences: www.amershambiosciences.com) and analysed by the Fragment Manager Program of DNAsis (MiraiBio Inc., Alameda, CA, USA).

\section{RT-PCR}

For expression analysis total RNA was isolated and reverse transcribed as described. ${ }^{23}$ Amplification of EFNB1 cDNA was performed by RT-PCR using primers 5'-GGCAGAG GAAGGCGAGGCGA-3' and 5'-GCAAGGGGAGGGGGT GTG-3' for amplification of a $1.2 \mathrm{~kb}$ product encompassing the entire coding region. Primer pair 5'-CAAGTTCCT GAGTGGGAAGG-3' and 5'-GTGTGGCCATCTTGACAG TG-3' was used to amplify a 455-bp product from exons 2-4, and primer pair 5'-ATCATGAAGGTTGGGCAAGA-3'

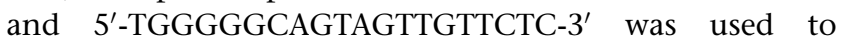
amplify a 467-bp product from exons 4 and 5. RT-PCR products of cells carrying heterozygous missense and frameshift mutations were cleaved with the respective restriction enzymes. RT-PCR products obtained from the c.407-2A > T EFNB1 allele were cloned into pGEM-Teasy vector (Promega: www.promega.com) and sequenced as described. ${ }^{11}$

\section{Real-time RT-PCR}

Quantitative RT-PCR analysis was performed using an $\mathrm{iQ}^{\mathrm{TM}}$ SYBR green supermix kit according to the manufacturer's instructions (Bio-Rad Laboratories $\mathrm{GmbH}$, München, Germany). Primers were the same as described above for the 455 bp EFNB1 RT-PCR product. Real-time amplification was carried out in an iCyler iQ Real-Time PCR Detection System (Bio-Rad Laboratories). To calculate the relative EFNB1 expression, the cDNA from housekeeping gene
G3PDH was amplified using primers described previously. ${ }^{23}$ The threshold cycle number $\left(C_{\mathrm{t}}\right)$ was measured using the iCycler and its associated software. Melting curves for each PCR were generated to ensure the purity of the amplification product. EFNB1 mRNA expression was normalised with $G 3 P D H$ mRNA expression by calculating $\Delta\left(\Delta C_{t}\right)$. Relative EFNB1 levels were calculated by the $2^{-\Delta\left(\Delta C_{\mathrm{t}}\right)}$ method. Results are given as fold differences to control primary fetal fibroblasts. ${ }^{24}$

\section{Results \\ EFNB1 expression in human cell cultures}

Primary human cell cultures established from patients' skin biopsies were analysed for EFNB1 expression by RT-PCR (Table 1). By the HUMAR assay for measuring the pattern of $\mathrm{X}$-inactivation in a given cell population, a random $\mathrm{X}$-inactivation was detected in the primary cell cultures derived from three CFNS patients (patient ID S16-S18, Table 1). In two further cell cultures derived from 1-yearold girls who underwent surgery because of coronal synostosis, a non-random X-inactivation pattern was observed (patient ID F3, S33, Table 1). Of the latter two cell cultures, only the one carrying missense mutation c.161C $>\mathrm{T}$ on the active X-chromosome (ID S33) was further analysed. One patient (ID 39) was not informative in the HUMAR assay.

\section{NMD of PTC-containing EFNB1 alleles}

Expression of wild-type and mutant EFNB1 transcripts was monitored by restriction enzyme cleavage of RT-PCR products. Transcripts from missense mutations c.409A $>\mathrm{G}$ and c.161C $>\mathrm{T}$ were readily detected in addition to the wild-type alleles (Figure 1). Mutations generating internal

Table 1 Summary of CFNS patients, EFNB1 mutations and EFNB1 mRNA expression

\begin{tabular}{|c|c|c|c|c|c|c|}
\hline Patient ID & $\$ 18$ & S33 & F3 & $S 17$ & $S 16$ & S39 \\
\hline Age (years) & 19 & 1 & 1 & 26 & 7 & 1 \\
\hline Classical CFND & + & + & + & + & + & + \\
\hline Additional features & None reported ${ }^{a}$ & $t^{b}$ & $t^{a, c}$ & $t^{a, d}$ & None reported ${ }^{a}$ & $++^{e}$ \\
\hline \multicolumn{7}{|l|}{ EFNB1 mutation } \\
\hline Nucleotide change & c. $409 \mathrm{~A}>\mathrm{G}$ & c. $161 \mathrm{C}>\mathrm{T}$ & c. $407-2 A>G$ & c. $407-2 \mathrm{~A}>\mathrm{T}$ & c.377_384del & c.614_615delCT \\
\hline Amino-acid change & p.T137A & p.P54L & Splice site mutation & Splice site mutation & Frameshift & p.S205fsX \\
\hline Exon/intron & 3 & 2 & 2 & 2 & 2 & 4 \\
\hline Restriction enzyme ${ }^{f}$ & SfaNl (+) & $B s / l(-)$ & Pstl (-) & Pstl $(-)$ & $\operatorname{Hinfl}(+)$ & Hinfl (-) \\
\hline X-inactivation in fibroblasts & Random & Non-random & Non-random & Random & Random & $\mathrm{ND}^{\mathrm{g}}$ \\
\hline EFNB1 mRNA & $w t+$ mutant & $w t+$ mutant & wt & $w t+\left(\right.$ mutant $\left.^{\mathrm{h}}\right)$ & $w t+\left(\right.$ mutant $\left.^{\mathrm{h}}\right)$ & $w t+$ mutant \\
\hline
\end{tabular}

a Patient description in reference Wieland et al. ${ }^{11}$

barge cleft lip and palate, coronal craniosynostosis.

${ }^{c}$ Coronal craniosynostosis, corpus callosum agenesis.

${ }^{\mathrm{d}}$ Duplex kidneys and ureters, duplication of the uterus, nail splitting.

eCoronal craniosynostosis, asymmetric thorax and legs, syndactyly III/IV of toes, duplex kidney, diaphragmatic hernia.

${ }^{\mathrm{f}}$ Mutation detection by a de novo occurring restriction enzyme site $(+)$ or by loss of a restriction enzyme site $(-)$.

${ }^{9}$ Could not be determined by the HUMAR assay.

${ }^{h}$ Very low amount of mutant transcript compared with wild-type transcript. 

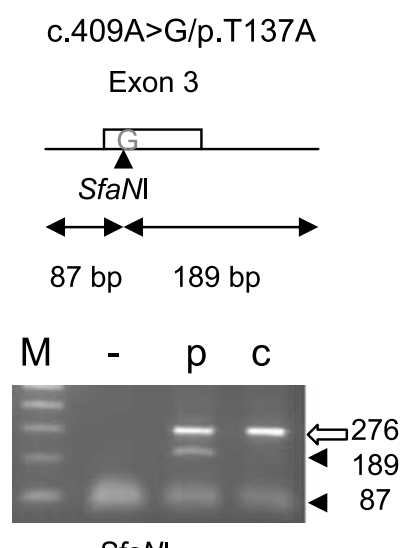

SfaNl
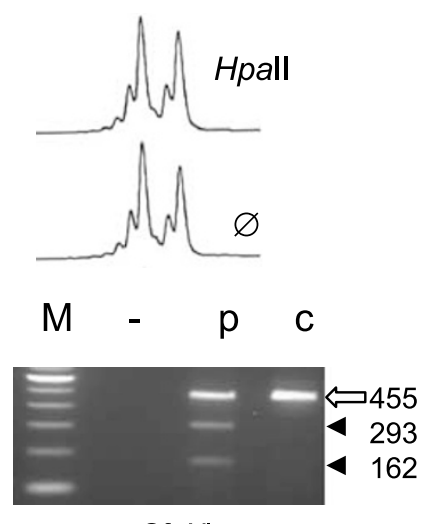

SfaNl

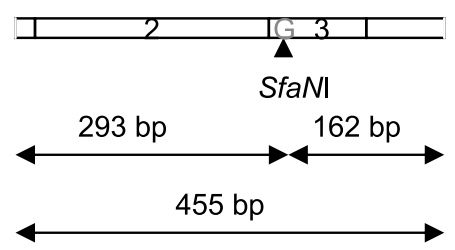

c. $161 \mathrm{C}>\mathrm{T} /$ p.P54L

Exon 2

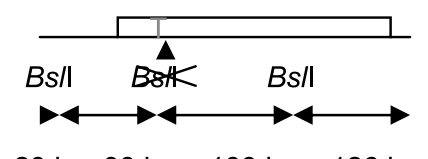

23 bp 98 bp 139 bp 126 bp

$M-p f m$

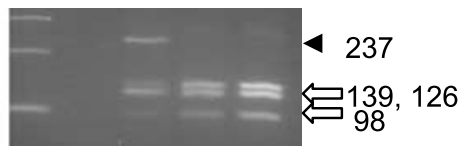

Bs/l

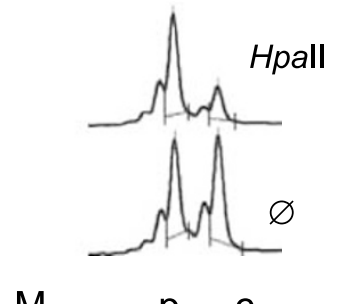

$M \quad-p \quad c$

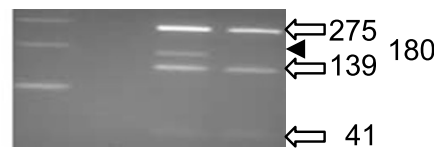

Bs/l

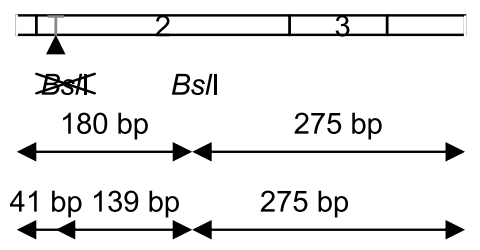

\section{G-PCR}

HUMARA

RT-PCR

Figure 1 Expression of ephrin-B1 gene (EFNB1) transcripts in primary fibroblasts harbouring heterozygous missense mutations c.409A $>\mathrm{G}$ (p.T137A) and c.161C > T (p.P54L), respectively. Mutations were verified by genomic PCR followed by restriction enzyme cleavage (G-PCR). The $\mathrm{X}$-inactivation status in fibroblast cultures was determined by human androgen receptor X-inactivation (HUMAR) assay. Expression of wild-type and mutant EFNB1 transcripts was determined by RT-PCR followed by restriction enzyme cleavage. DNA and RNA from the patients (lanes p), their fathers (lane f), mothers (lane $\mathrm{m}$ ) or a control person (lanes $\mathrm{c}$ ) were analysed. Wild-type alleles are indicated by open arrows, mutant alleles are indicated by filled arrow heads. Size markers are shown in lanes M (100 bp DNA ladder, Invitrogen) and PCRs without template DNA in lanes (-).

PTCs were splice site mutation c.407-2A $>$ T and frameshift mutation c.377_384delTCAAGAAG (Figure 2). Splice site mutation c.407-2A $>\mathrm{T}$ was predicted by computationalbased analysis to result in either a larger sized RT-PCR product of $1.2 \mathrm{~kb}$ including intron 2 or a smaller sized RT-PCR product of $362 \mathrm{bp}$ generated by skipping of exon 3 (-93 bp). Skipping of exon 3 does not change the reading frame and would allow production of a structurally distorted transmembrane ephrin-B1 protein. By RT-PCR, the $1.2 \mathrm{~kb}$ amplicon was detected in addition to the 455-bp wild-type allele (Figure 3a). However, the amount of the $1.2 \mathrm{~kb}$ product was drastically reduced in comparison to the wild-type product. Cloning and sequencing of this $1.2-\mathrm{kb}$ amplicon revealed retention of intron 2 in the transcript (Figure 2). The corresponding amplicon expected from exon 3 skipping was not observed. Distinction of wild-type and mutant c.377_384delTCAAGAAG RT-PCR products was done by Hinfl cleavage (Figure 3a). The wild-type PCR product of $455 \mathrm{bp}$ and two cleavage fragments of 252 and $195 \mathrm{bp}$ from the mutant amplicon were predicted. However, only the wild-type PCR product but not the amplicon from the mutation allele was detected. This suggests that PTCs located in exon 2 (frameshift mutation c.377_384delTCAAGAAG) and in intron 2 (splice site 

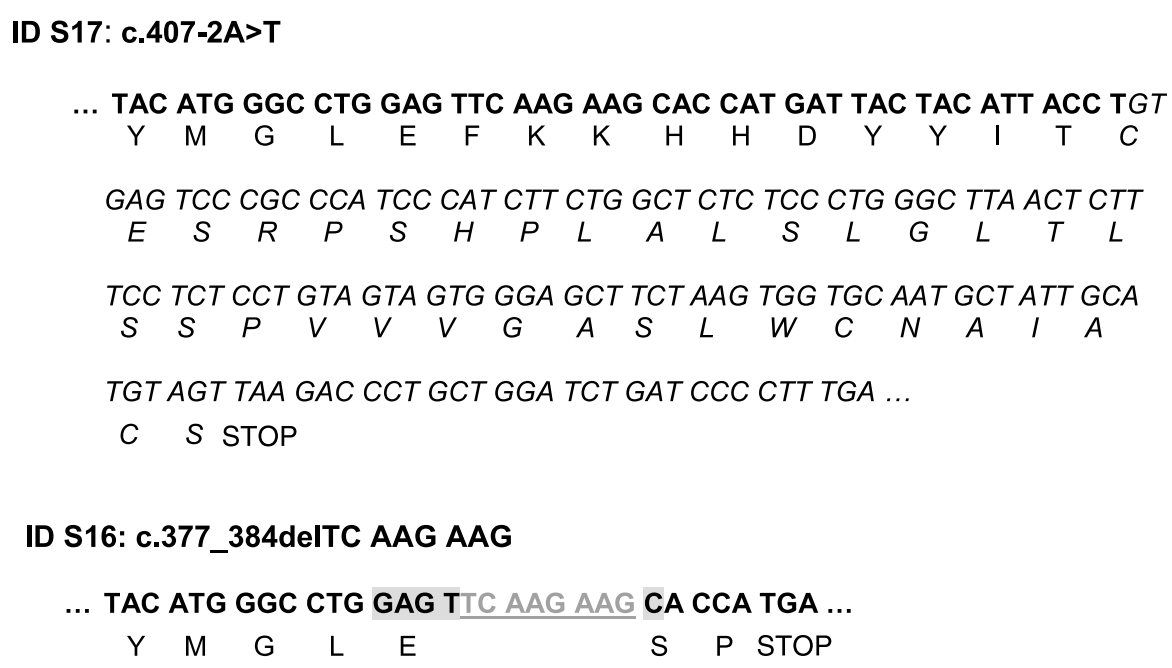

ID S39: c.614_615delCT

... GGC TCC CTG GGT GAC TCT GAT GGC AAG CAT C...

G S L G D STOP

Figure 2 Sequences of the mutations generating premature termination codons (PTCs) in ephrin-B1 gene (EFNB1). Mutation c.407-2A $>$ T at the splice acceptor site of exon 2 causes retention of intron 2. This creates an internal PTC located 36 codons downstream of the exon 2 junction site. Sequence of exon 2 is shown in bold, the $5^{\prime}$ part of intron 2 is shown in italic letters. Frameshift mutation c.377_384del causes a first PTC located three codons downstream of the $8 \mathrm{bp}$ deletion in exon 2. Sequence of exon 2 is shown in bold letters, the deleted 8 bp are underlined and the novel Hinfl site is highlighted. Frameshift mutation c.614_615delCT generates a PTC. It appears 11 nucleotides upstream of the exon 4 junction site. Sequence of exon 4 is shown in bold letters, the deleted $2 \mathrm{bp}$ are underlined, the novel stop codon TGA is set in grey, and the Hinfl site is highlighted.

mutation c.407-2A $>$ T) cause EFNB1 transcript depletion by NMD. In contrast to the mutations resulting in internal PTCs, frameshift mutation c.614_615delCT generates a PTC just 11 nucleotides upstream of the 3 -end of the penultimate exon 4 that may cause mRNA to escape NMD (Figure 2). Analysis of primary and subcultured patient cells clearly showed expression of the c.614_615delCT mutant transcripts as was expected (Figure $3 b$ ).

Clonal expansion of cells expressing wild-type or mutant EFNB1: separation of the cellular mosaic

We established subcultures of the primary cell culture from CFNS patient S16 harbouring frameshift mutation c.377_del384del. Clonality of the subcultures was proven by the HUMAR assay. In contrast to the primary cell culture exhibiting a random X-inactivation pattern (as shown in Figure 3a), cell clones Ks1 and Ks20 showed an exclusive inactivation of either X-chromosome (Figure 4a). To evaluate the relative expression of EFNB1 transcripts in these cells, real-time quantitative RT-PCR was performed. Compared to control primary fetal fibroblasts, EFNB1 was expressed 12-fold less in primary skin fibroblasts and subclone Ks1 obtained from patient S16. However, clone Ks20 with the mutant EFNB1 allele on the active X-chromosome showed a 500-fold reduced EFNB1 transcript level compared to primary fetal fibroblasts (Figure 4a). To investigate whether residual EFNB1 transcripts from the mutant allele are detectable in Ks20, exhaustive RT-PCR amplification ( $\geq 42$ cycles) combined with HinfI restriction enzyme digestion was performed. By this assay fragments of 252 and $195 \mathrm{bp}$ derived from the mutant transcripts were finally detected. Additional faint bands observed at the size for the wild-type allele ( $455 \mathrm{bp}$ ) may be due to incomplete HinfI cleavage or, may be expressed at very low levels from the wild-type EFNB1 allele located on the inactive $\mathrm{X}$-chromosome in clone Ks20. The clonal separation of wild-type and mutant EFNB1-expressing cells not only led us to analyse the expression of a mutant PTC-containing allele, but also shows that heterozygous CFNS patients indeed carry two distinct cell populations with respect to EFNB1 gene function. Since PTCs in internal exons elicit $\mathrm{NMD}$, we further tested whether the protein synthesis inhibitor cycloheximide was able to reverse cytoplasmic NMD. ${ }^{25}$ However, treatment of the cells with up to $1 \mathrm{mg} / \mathrm{ml}$ cycloheximide for $2 \mathrm{~h}$ did not result in substantial upregulation of internal PTC-containing EFNB1 transcripts (Figure 4b).

\section{Discussion}

In human fibroblasts, expression of EFNB1 has been previously described as part of a comprehensive $\mathrm{X}$-inactivation profile of the human X-chromosome. ${ }^{7}$ 
a c. $407-2 \mathrm{~A}>\mathrm{T}$
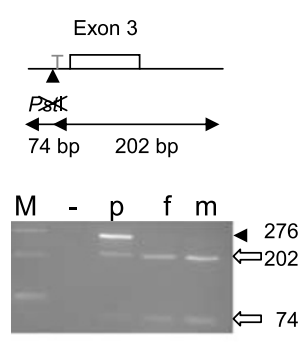

Pstl
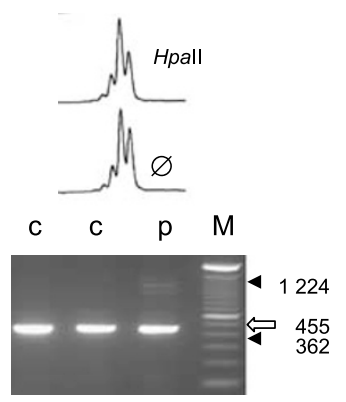

$\varnothing$

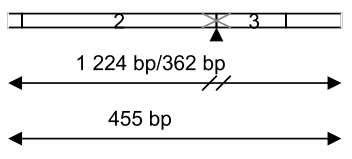

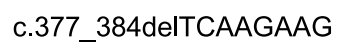
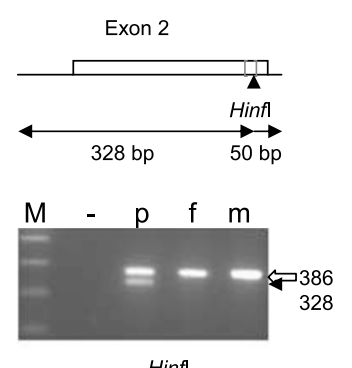

Hinfl

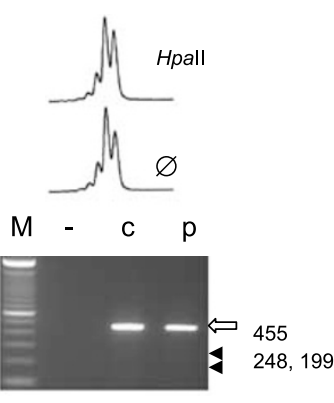

Hinfl

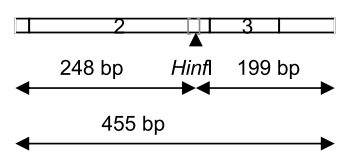

b c.614_615delCT

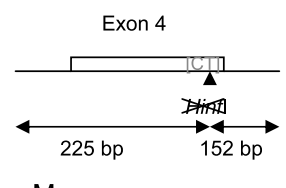

$M-p \quad c$

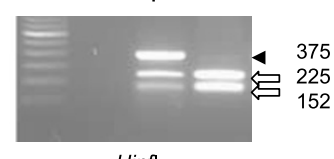

Hinfl

HUMARA
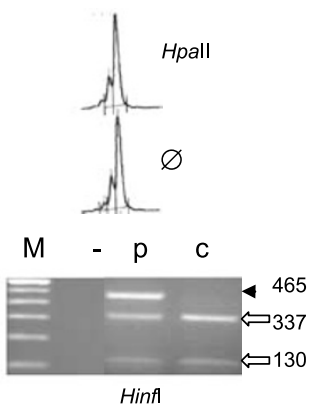

\section{RT-PCR}

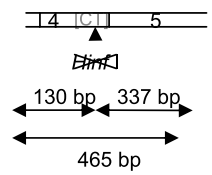

G-PCR

HUMARA

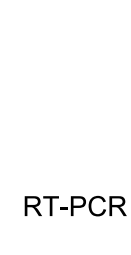

Figure 3 Expression of ephrin-B1 gene (EFNB1) transcripts in primary patient fibroblasts harbouring heterozygous premature termination codon (PTC) mutations. (a) Analysis of splice site mutation c.407-2 > T and frameshift mutation c.377_384delTCAAGAAG. (b) Analysis of frameshift mutation C.614_615delCT. Genomic mutations were verified by PCR and restriction enzyme cleavage (G-PCR). The X-inactivation status in fibroblast cultures was determined by human androgen receptor X-inactivation (HUMAR) assay. Wild-type and mutant EFNB1 transcripts were determined by RT-PCR followed by restriction enzyme cleavage. DNA and RNA from the patients (lanes $\mathrm{p}$ ), their fathers (lanes $\mathrm{f}$ ), mothers (lanes $\mathrm{m}$ ) or controls (lanes $\mathrm{c}$ ) were analysed. Wild-type alleles are indicated by open arrows, mutant alleles are indicated by filled arrow heads. Size markers are shown in lanes $\mathrm{M}$ (100 bp DNA ladder, Invitrogen) and PCRs without template DNA in lanes (-).

In these fibroblasts established from females, the EFNB1 gene is subject to random $\mathrm{X}$-inactivation. A random $\mathrm{X}$-inactivation was also observed in three of the five primary fibroblast cultures established from the biopsies available from CFNS patients known to be heterozygous for EFNB1 mutations. A non-random pattern of X-inactivation was present in fibroblast cultures from two patients. This was probably due to cell clonality in the biopsies, since a random X-inactivation was detected in peripheral blood cells of these patients. To assess the effects of various mutations on EFNB1 mRNA levels, expression of EFNB1 transcripts was studied by RT-PCR. Missense mutation c.161C > T (p.P54L) has been recurrently detected in two sporadic CFNS patients and two CFNS families. ${ }^{4,11,12}$ Missense mutation c.409A $>$ G (p.T137A) has been previously described in a sporadic patient. ${ }^{11}$ Both mutant alleles showed about equal amounts of transcript compared to the wild-type allele in primary fibroblast cultures exhibiting a random or nearly random X-inactivation. Since these mutants demarcate the ends of the $\beta$-strands B and $\mathrm{H}$ in the extracellular ephrin domain, they are unlikely to result in unstable proteins. Rather, these CFNS-causing mutations are likely to disturb the formation of higherorder ligand receptor clusters at the sites of cell-cell contact. $^{26}$

In contrast to the missense mutation alleles, severe depletion of transcripts was observed for the mutant alleles harbouring either splice site mutation c.407-2A $>\mathrm{T}$ at the exon 2/3 boundary or frameshift mutation c.377_384del. Splice site mutations at the exon $2 / 3$ boundary have been described in five independent CFNS patients. ${ }^{9,11,12}$ Although splice site mutations at the exon $2 / 3$ junction could cause skipping of exon 3 without changing the reading frame of the remaining two exons of EFNB1, our results support retention of intron 2 being favoured over inappropriate exon 3 skipping. Retention of intron 2 produces numerous PTCs, the first occurring 36 codons downstream of the exon 2 boundary. Similarly, frameshift mutation c.377_384del produces numerous PTCs, the first occurring just three codons downstream of the 8-bp 


\section{RT-PCR}

$M-K s 1 K s 20$

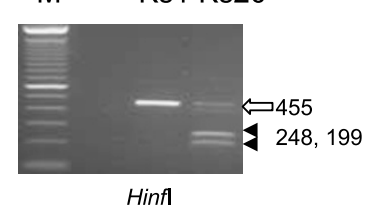

Hinfl

b $c .407-2 A>T$

Cycloheximide $[\mathrm{mg} / \mathrm{ml}]$

\section{HUMARA}

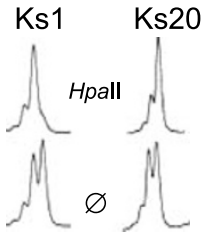

$\varnothing 5$
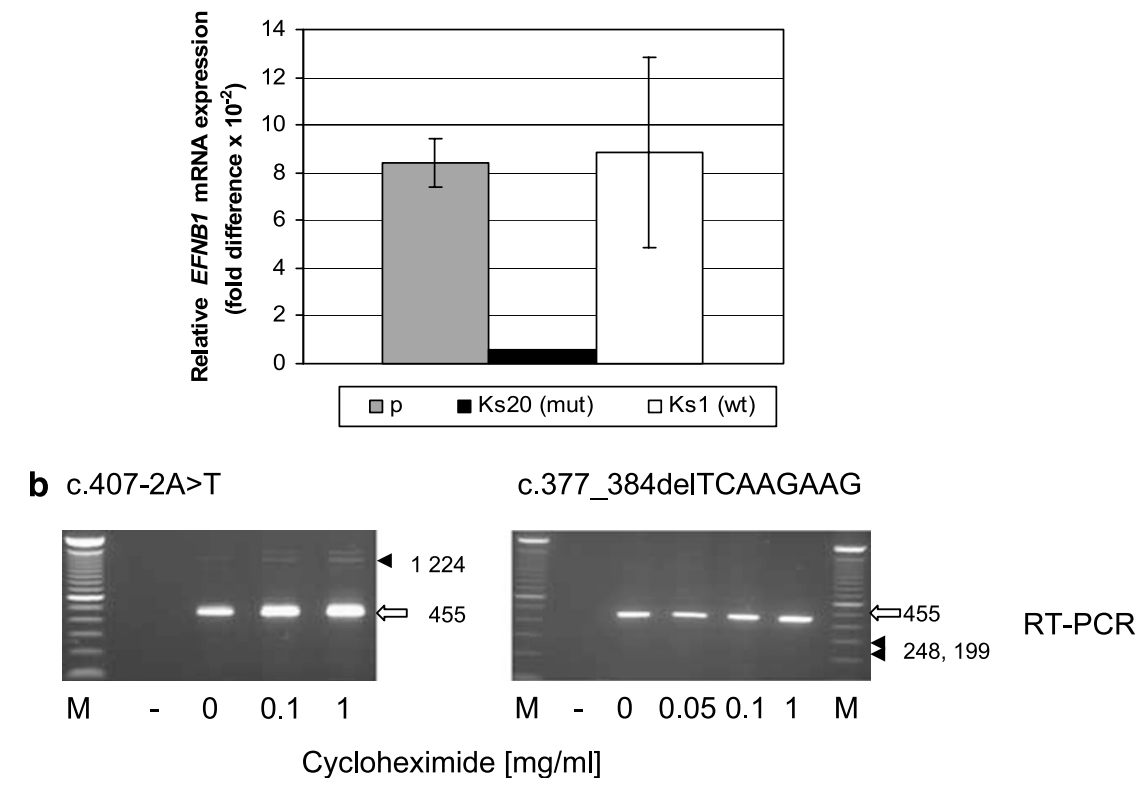

Figure 4 Cell culture treatment of primary patient fibroblasts. (a) Analysis of ephrin-B1 gene (EFNB1) transcripts expressed in clonal descendants of primary fibroblasts. Cell clones Ks 1 and Ks20 showed a skewed X-inactivation pattern corresponding to the inactivation of either the paternal or maternal X-chromosome (upper panel). RT-PCR followed by Hinf/ cleavage revealed expression of wild-type EFNB1 transcripts in Ks1 and very low amounts of mutant EFNB1 transcripts in Ks20. The relative EFNB1 expression in the primary fibroblasts, clonal descendants Ks1 and Ks20, was measured by real-time RT-PCR (lower panel). (b) Cycloheximide treatment of primary fibroblasts harbouring the heterozygous splice site mutation c.407-2 $>$ T or frameshift mutation c.377_384delTCAAGAAG showed no substantial effect on expression of the premature termination codon (PTC)-containing EFNB1 transcripts. The wild-type allele is indicated by an open arrow, fragments of the mutant allele are indicated by filled arrow heads. Size markers (lanes M; 100 bp DNA ladder, Invitrogen) and PCR without template DNA (lanes -) are indicated accordingly.

deletion. Previously, it has been shown for several diseasecausing genes that NMD occurs when a nonsense codon resides >50-55 nucleotides upstream of a splice generating exon-exon junction. ${ }^{18,20}$ Both of the internal truncating EFNB1 mutations investigated satisfy these requirements to trigger decay of the mutant transcripts by the NMD pathway. In contrast to internal truncating mutations, frameshift mutation c.614_615delCT generates the first PTC 11 nucleotides upstream of the junction of exons 4 and 5, which disobeys the $>50-55 \mathrm{bp}$ rule. Because the NMD surveillance system does not differentiate nonsense codon mutations in the last and the $3^{\prime}$-end of the exon before it from the normal termination codon, such transcripts may escape NMD. Apparently, this applies to frameshift mutation c.614_615delCT. Whether the observed transcripts will give rise to a truncated protein product, and whether such truncated soluble ephrin-B1 ligands exhibit dominant-negative or gain-of-function effects still needs be investigated. In this context, it is remarkable that truncating mutations in exons 4 and 5 of EFNB1 appear to be rather infrequent in CFNS. To date, five CFNS patients have been described with truncating mutations. ${ }^{11,12,14}$ The clinical phenotypes appear to be similar to the phenotypes in other patients with the exception of diaphragmatic hernia, which has been observed in CFNS males and solely in two CFNS females harbouring putative ephrin-B1 truncating mutations.

Clonal expansions from primary fibroblasts of a heterozygous CFNS patient showed that it is possible to separate the wild-type and mutant cellular phenotypes in cell culture. This supports the concept of cellular interference of wild-type and mutant cell populations to be the cause 
for severe disease manifestation in CFNS females. In an in vitro assay with zebrafish embryos, the cellular behaviour of Eph receptor and ephrin ligand expressing adjacent cell populations was previously studied. ${ }^{27}$ This showed that only a few cells migrated into the adjacent territory, whereas extensive cell intermingling occurred when ephrin was omitted from one of the two cell populations. A similar situation may appear in CFNS when wild-type and mutant EFNB1-expressing cell clones expand during developmental processes. Such a situation does not occur in hemizygous obligate carrier males and accordingly, CFNS males are mildly affected. Somatic mosaicism, however, might manifest in males similar to CFNS in females, since this could lead to cellular interference analogous to what occurs through random $\mathrm{X}$-inactivation in a heterozygous state. ${ }^{12}$ The first, and so far only, male mosaic identified with an EFNB1 mutation had a low level of mosaicism in blood (3\%) and hair roots (2\%) as quantified by pyrosequencing, but he presented with mild features of the CFNS male-carrier state except hypertelorism. It appears quite surprising that a mutant ratio of less than $1 / 10$ in a male mosaic already suffices to cause a mild CFNS phenotype. This mild phenotype may well be caused by a low degree of cellular interference. We expect a more severe phenotype in a male mosaic with a wild-type to mutant ratio of close to 1 as it occurs in heterozygous CFNS females.

\section{Acknowledgements}

We thank the patients and their families and the clinicians for their cooperation. We thank S Engelberg, G Koch and N Reissner for their technical assistance. Dr Reardon gratefully cites the support of the Children's Medical and Research Foundation at Our Lady's Hospital for Sick Children. RM is a recipient of a PhD scholarship from the Graduate Program of the Deutsche Forschungsgemeinschaft (DFGGRK 1167).

\section{References}

1 Wieacker P, Wieland I: Clinical and genetic aspects of craniofrontonasal syndrome: towards resolving a genetic paradox. $\mathrm{Mol}$ Gen Metab 2005; 86: 110-116.

2 Feldman GJ, Ward DE, Lajeunie-Renier E et al: A novel phenotypic pattern in X-linked inheritance: craniofrontonasal syndrome maps to Xp22. Hum Mol Genet 1997; 6: 1937-1941.

3 Wieland I, Jakubiczka S, Muschke P et al: Mapping of a further locus for X-linked craniofrontonasal syndrome. Cytogenet Genome Res 2002; 99: 285-288.

4 Wieland I, Jakubiczka S, Muschke P et al: Mutations of the ephrinB1 gene cause craniofrontonasal syndrome. Am J Hum Genet 2004; 74: 1209-1215.

5 Yamaguchi Y, Pasquale EB: Eph receptors in the adult brain. Curr Opin Neurobiol 2004; 14: 288-296.

6 Klein R: Eph/ephrin signaling in morphogenesis, neural development and plasticity. Curr Opin Cell Biol 2004; 16: 580-589.
7 Carrel L, Willard HF: X-inactivation profile reveals extensive variability in X-linked gene expression in females. Nature 2005; 434: 400-404.

8 Compagni A, Logan M, Klein R, Adams RH: Control of skeletal patterning by ephrinB1-EphB interactions. Dev Cell 2003; 5: $217-230$.

9 Twigg SRF, Kan R, Babbs C et al: Mutations of ephrin-B1 (EFNB1), a marker of tissue boundary formation, cause craniofrontonasal syndrome. Proc Natl Acad Sci USA 2004; 101: 8652-8657.

10 Davy A, Aubin J, Soriano P: Ephrin-B1 forward and reverse signaling are required during mouse development. Genes Dev 2004; 18: 572-583.

11 Wieland I, Reardon W, Jakubiczka S et al: Twenty-six novel EFNB1 mutations in familial and sporadic craniofrontonasal syndrome (CFNS). Hum Mutat 2005; 26: 113-118.

12 Twigg SRF, Matsumoto K, Kidd AMJ et al: The origin of EFNB1 mutations in craniofrontonasal syndrome: frequent mosaicism and explanation of the paucity of carrier males. Am J Hum Genet 2006; 78: 999-1010.

13 Wieland I, Weidner C, Ciccone R et al: Contiguous gene deletions involving EFNB1, OPHN1, PJA1 and EDA in patients with craniofrontonasal syndrome (CFNS). Clin Genet 2007; 72: 506-516.

14 Shotelersuk V, Siriwan P, Ausavarat S: A novel mutation in EFNB1, probably with a dominant negative effect, underlying craniofrontonasal syndrome. Cleft Palate Craniofac J 2006; 43: 152-154.

15 Krull CE, Lansford R, Gale NW et al: Interactions of Eph-related receptors and ligands confer rostrocaudal pattern to trunk neural crest migration. Curr Biol 1997; 7: 571-580.

16 Koblar SA, Krull CE, Pasquale EB et al: Spinal motor axons and neural crest cells use different molecular guides for segmental migration through the rostral half-somite. J Neurobiol 2000; 42: $437-447$.

17 Santiago A, Erickson CA: Ephrin-B ligands play a dual role in the control of neural crest cell migration. Development 2002; 129: $3621-3632$.

18 Frischmeyer PA, Dietz HC: Nonsense-mediated mRNA decay in health and disease. Hum Mol Genet 1999; 8: 1893-1900.

19 Holbrook JA, Neu-Yilik G, Hentze MW, Kulozik AE: Nonsensemediated decay approaches the clinic. Nat Genet 2004; 36: $801-808$.

20 Lejeune F, Maquat LE: Mechanistic links between nonsensemediated mRNA decay and pre-mRNA splicing in mammalian cells. Curr Opin Cell Biol 2005; 17: 309-315.

21 Inoue $\mathrm{K}$, Khajavi $\mathrm{M}$, Ohyama $\mathrm{T}$ et al: Molecular mechanism for distinct neurological phenotypes conveyed by allelic truncating mutations. Nat Genet 2004; 36: 361-369.

22 Allen RC, Zoghbi HY, Moseley AB, Rosenblatt HM, Belmont JW: Methylation of HpaII and HhaI sites near the polymorphic CAG repeat in the human androgen-receptor gene correlates with $\mathrm{X}$ chromosome inactivation. Am J Hum Genet 1992; 51: 1229-1239.

23 Wieland I, Arden KC, Michels D et al: Isolation of DICE1: a gene frequently affected by $\mathrm{LOH}$ and downregulated in lung carcinomas. Oncogene 1999; 18: 4530-4537.

24 Livak KJ, Schmittgen TW: Analysis of relative gene expression data using real-time quantitative PCR and the $2^{-\Delta(\Delta C \mathrm{t})}$ method. Methods 2001; 25: 402-408.

25 Carter MS, Doskow J, Morris P et al: A regulatory mechanism that detects premature nonsense codons in T-cell receptor transcripts in vivo is reversed by protein synthesis inhibitors in vitro. J Biol Chem 1995; 270: 28995-29003.

26 Nikolov DB, Li C, Barton WA, Himanen J-P: Crystal structure of the ephrin-B1 ectodomain: Implications for receptor recognition and signaling. Biochemistry 2005; 44: 10947-10953.

27 Mellitzer G, Xu Q, Wilkinson DG: Eph receptors and ephrins restrict cell intermingling and communication. Nature 1999; 400: $77-81$. 